\title{
COMMON FIXED POINT THEOREMS OF CONTRACTIVE-TYPE MAPPINGS
}

\section{HEE SOO PARK and JEONG SHEOK UME}

\author{
Received 10 April 2004
}

\begin{abstract}
Using the concept of $D$-metric we prove some common fixed point theorems for generalized contractive mappings on a complete $D$-metric space. Our results extend, improve, and unify results of Fisher and Ćirić.
\end{abstract}

2000 Mathematics Subject Classification: 47H10.

1. Introduction. The Banach contraction mapping principle is well known. There are many generalizations of that principle to single- and multivalued mappings (see $[1,4,5,10,11,12])$. The study of maps satisfying some contractive conditions has been the center of rigorous research activity since such mappings have many applications (see [2, 3, 9, 13, 14, 15]).

In 1998, Ćirić [6] proved a common fixed point theorem for nonlinear mappings on a complete metric space: let $(X, d)$ be a complete metric space and $S, T: X \rightarrow X$ selfmaps such that $d(S T x, T S y) \leq \max \left\{\varphi_{1}[(1 / 2)(d(x, S y)+d(y, T x))], \varphi_{2}[d(x, T x)]\right.$, $\left.\varphi_{3}[d(y, S y)], \varphi_{4}[d(x, y)]\right\}$ for all $x, y$ in $X$, where $\varphi_{i} \in \Phi(i=1,2,3,4)$. If $S$ or $T$ is continuous, then $S$ and $T$ have a unique common fixed point. This result improved and extended a theorem of Fisher [8].

In this paper, using the concept of $D$-metric, we prove common fixed point theorems which extend, improve, and unify the corresponding theorems of Fisher [8] and Ćirić [6].

Throughout the paper, by $\Phi$ we denote the collection of functions $\varphi:[0, \infty) \rightarrow[0, \infty)$ which are continuous from the right, nondecreasing, and which satisfy the condition $\varphi(t)<t$ for all $t>0$. We denote by $\mathbb{N}$ the set of all positive integers.

2. Preliminaries. Before proving the main theorem, we will introduce some definitions and lemmas.

Definition 2.1 [7]. Let $X$ be any nonempty set. A $D$-metric for $X$ is a function $D: X \times X \times X \rightarrow R$ such that

(1) $D(x, y, z) \geq 0$ for all $x, y, z \in X$ and equality holds if and only if $x=y=z$,

(2) $D(x, y, z)=D(x, z, y)=D(y, x, z)=D(y, z, x)=D(z, x, y)=D(z, y, x)$ for all $x, y, z \in X$,

(3) $D(x, y, z) \leq D(x, y, a)+D(x, a, z)+D(a, y, z)$ for all $x, y, z \in X$.

If $D$ is a $D$-metric for $X$, then the ordered pair $(X, D)$ is called a $D$-metric space or the set $X$, together with a $D$-metric, is called a $D$-metric space. We note that to 
a given ordinary metric space $(X, d)$ there corresponds a $D$-metric space $(X, D)$, but the converse may not be true (see Example 3.3). In this sense the $D$-metric spaces are the generalizations of the ordinary metric space.

DEFINITION 2.2 [7]. A sequence $\left\{x_{n}\right\}$ of points of a $D$-metric space $X$ converges to a point $x \in X$ if for an arbitrary $\varepsilon>0$, there exists an $n_{0} \in \mathbb{N}$ such that for all $n>m \geq n_{0}$, $D\left(x_{m}, x_{n}, x\right)<\varepsilon$.

Definition 2.3 [7]. A sequence $\left\{x_{n}\right\}$ of points of a $D$-metric space $X$ is said to be a $D$-Cauchy sequence if for an arbitrary $\varepsilon>0$, there exists an $n_{0} \in \mathbb{N}$ such that for all $p>n>m \geq n_{0}, D\left(x_{m}, x_{n}, x_{p}\right)<\varepsilon$.

Definition 2.4 [7]. A $D$-metric space $X$ is a complete $D$-metric space if every $D$ Cauchy sequence $\left\{x_{n}\right\}$ in $X$ converges to a point $x$ in $X$.

Definition 2.5. A real-valued function $f$ defined on a metric space $X$ is said to be lower semicontinuous at a point $t$ in $X$ if $\lim _{x \rightarrow t} \inf f(x)=\infty$ or $\lim _{x \rightarrow t} \inf f(x) \geq f(t)$.

Definition 2.6. A real-valued function $f$ defined on a metric space $X$ is said to be upper semicontinuous at a point $t$ in $X$ if $\lim _{x \rightarrow t} \sup f(x)=\infty$ or $\lim _{x \rightarrow t} \sup f(x) \leq f(t)$.

Definition 2.7. Let $x_{0} \in X$ and $\varepsilon>0$ be given. Then the open ball $B\left(x_{0}, \varepsilon\right)$ in $X$ centered at $x_{0}$ of radius $\varepsilon$ is defined by

$$
B\left(x_{0}, \varepsilon\right)=\left\{y \in X \mid D\left(x_{0}, y, y\right)<\varepsilon \text { if } y=x_{0}, \sup _{z \in X} D\left(x_{0}, y, z\right)<\varepsilon \text { if } y \neq x_{0}\right\}
$$

Then the collection of all open balls $\{B(x, \varepsilon): x \in X\}$ defines the topology on $X$ denoted by $\tau$.

Lemma 2.8 [7]. The $D$-metric for $X$ is a continuous function on $X \times X \times X$ in the topology $\tau$ on $X$.

LEMmA 2.9 [6]. If $\varphi_{1}, \varphi_{2} \in \Phi$, then there is some $\varphi \in \Phi$ such that $\max \left\{\varphi_{1}(t)\right.$, $\left.\varphi_{2}(t)\right\} \leq \varphi(t)$ for all $t>0$.

LEMMA 2.10. Let $(X, D)$ be a $D$-metric space. Let $g: X \times X \rightarrow X$ be a mapping and let $S, T: X \rightarrow X$ be mappings such that

$$
\begin{aligned}
& \max \{D(S T x, T S y, g(S T x, T S y)), D(T S y, S T x, g(T S y, S T x))\} \\
& \leq \max \{ \varphi_{1}\left[\frac{1}{2}(D(x, S y, g(x, S y))+D(y, T x, g(y, T x)))\right], \\
& \varphi_{2}[D(x, T x, g(x, T x))], \varphi_{3}[D(y, S y, g(y, S y))], \\
&\left.\varphi_{4}[D(x, y, g(x, y))]\right\}
\end{aligned}
$$

for all $x, y \in X$, where $\varphi_{i} \in \Phi(i=1,2,3,4)$,

$$
x=y \Longrightarrow D(x, y, g(x, y))=0
$$


and

$$
\begin{gathered}
\max \{D(x, z, g(x, z)), D(x, y, g(x, z)), D(y, z, g(x, z))\} \\
\leq D(x, y, g(x, y))+D(y, z, g(y, z))
\end{gathered}
$$

for all $x, y, z \in X$. The sequence $\left\{x_{n}\right\}$ is defined by $x_{0} \in X, x_{2 n+1}=T x_{2 n}$, and $x_{2 n+2}=$ $S x_{2 n+1}$ for every $n \in \mathbb{N} \cup\{0\}$. Then

(I) for an arbitrary $\varepsilon>0$, there exists a positive integer $L$ such that $L \leq n<m$ implies $\max \left\{D\left(x_{n}, x_{m}, g\left(x_{n}, x_{m}\right)\right), D\left(x_{m}, x_{n}, g\left(x_{m}, x_{n}\right)\right)\right\}<\varepsilon$,

(II) a sequence $\left\{x_{n}\right\}_{n=0}^{\infty}$ is a D-Cauchy sequence.

Proof. Let $M=\max \left\{D\left(x_{0}, x_{1}, g\left(x_{0}, x_{1}\right)\right), D\left(x_{1}, x_{2}, g\left(x_{1}, x_{2}\right)\right), D\left(x_{2}, x_{1}, g\left(x_{2}, x_{1}\right)\right)\right\}$. Since all $\varphi_{i}$ are nondecreasing functions by (2.2), (2.3), and (2.4),

$$
\begin{aligned}
\max \{ & \left\{\left(x_{2}, x_{3}, g\left(x_{2}, x_{3}\right)\right), D\left(x_{3}, x_{2}, g\left(x_{3}, x_{2}\right)\right)\right\} \\
= & \max \left\{D\left(S T x_{0}, T S x_{1}, g\left(S T x_{0}, T S x_{1}\right)\right), D\left(T S x_{1}, S T x_{0}, g\left(T S x_{1}, S T x_{0}\right)\right)\right\} \\
\leq & \max \left\{\varphi_{1}\left[\frac{1}{2}\left(D\left(x_{0}, S x_{1}, g\left(x_{0}, S x_{1}\right)\right)+D\left(x_{1}, T x_{0}, g\left(x_{1}, T x_{0}\right)\right)\right)\right],\right. \\
& \quad \varphi_{2}\left[D\left(x_{0}, T x_{0}, g\left(x_{0}, T x_{0}\right)\right)\right], \varphi_{3}\left[D\left(x_{1}, S x_{1}, g\left(x_{1}, S x_{1}\right)\right)\right], \\
& \left.\quad \varphi_{4}\left[D\left(x_{0}, x_{1}, g\left(x_{0}, x_{1}\right)\right)\right]\right\} \\
\leq & \max \left\{\varphi_{1}(M), \varphi_{2}(M), \varphi_{3}(M), \varphi_{4}(M)\right\} \\
\leq & \varphi(M),
\end{aligned}
$$

where $\varphi \in \Phi$. Such $\varphi$ exists from an extended version of Lemma 2.9. Therefore, we have $\max \left\{D\left(x_{2}, x_{3}, g\left(x_{2}, x_{3}\right)\right), D\left(x_{3}, x_{2}, g\left(x_{3}, x_{2}\right)\right)\right\} \leq \varphi(M)$. Again, from (2.2), (2.3), and (2.4), we get

$$
\begin{aligned}
\max \{ & \left\{\left(x_{3}, x_{4}, g\left(x_{3}, x_{4}\right)\right), D\left(x_{4}, x_{3}, g\left(x_{4}, x_{3}\right)\right)\right\} \\
= & \max \left\{D\left(T S x_{1}, S T x_{2}, g\left(T S x_{1}, S T x_{2}\right)\right), D\left(S T x_{2}, T S x_{1}, g\left(S T x_{2}, T S x_{1}\right)\right)\right\} \\
\leq & \max \left\{\varphi_{1}\left[\frac{1}{2}\left(D\left(x_{2}, S x_{1}, g\left(x_{2}, S x_{1}\right)\right)+D\left(x_{1}, T x_{2}, g\left(x_{1}, T x_{2}\right)\right)\right)\right],\right. \\
& \quad \varphi_{2}\left[D\left(x_{2}, T x_{2}, g\left(x_{2}, T x_{2}\right)\right)\right], \varphi_{3}\left[D\left(x_{1}, S x_{1}, g\left(x_{1}, S x_{1}\right)\right)\right], \\
& \left.\quad \varphi_{4}\left[D\left(x_{2}, x_{1}, g\left(x_{2}, x_{1}\right)\right)\right]\right\} \\
\leq & \max \left\{\varphi_{1}(M), \varphi_{2}[\varphi(M)], \varphi_{3}(M), \varphi_{4}(M)\right\} \\
\leq & \varphi(M) .
\end{aligned}
$$

Using the obtained relations $\max \left\{D\left(x_{2}, x_{3}, g\left(x_{2}, x_{3}\right)\right), D\left(x_{3}, x_{2}, g\left(x_{3}, x_{2}\right)\right)\right\} \leq \varphi(M)$ and $\max \left\{D\left(x_{3}, x_{4}, g\left(x_{3}, x_{4}\right)\right), D\left(x_{4}, x_{3}, g\left(x_{4}, x_{3}\right)\right)\right\} \leq \varphi(M)$, from (2.2), (2.3), and (2.4), 
we get

$$
\begin{aligned}
\max \{ & \left\{\left(x_{4}, x_{5}, g\left(x_{4}, x_{5}\right)\right), D\left(x_{5}, x_{4}, g\left(x_{5}, x_{4}\right)\right)\right\} \\
= & \max \left\{D\left(S T x_{2}, T S x_{3}, g\left(S T x_{2}, T S x_{3}\right)\right), D\left(T S x_{3}, S T x_{2}, g\left(T S x_{3}, S T x_{2}\right)\right)\right\} \\
\leq & \max \left\{\varphi_{1}\left[\frac{1}{2}\left(D\left(x_{2}, S x_{3}, g\left(x_{2}, S x_{3}\right)\right)+D\left(x_{3}, T x_{2}, g\left(x_{3}, T x_{2}\right)\right)\right)\right],\right. \\
& \varphi_{2}\left[D\left(x_{2}, T x_{2}, g\left(x_{2}, T x_{2}\right)\right)\right], \varphi_{3}\left[D\left(x_{3}, S x_{3}, g\left(x_{3}, S x_{3}\right)\right)\right], \\
& \left.\varphi_{4}\left[D\left(x_{2}, x_{3}, g\left(x_{2}, x_{3}\right)\right)\right]\right\} \\
\leq & \max \left\{\varphi_{1}[\varphi(M)], \varphi_{2}[\varphi(M)], \varphi_{3}[\varphi(M)], \varphi_{4}[\varphi(M)]\right\} \\
\leq & \varphi^{2}(M) .
\end{aligned}
$$

Similarly, again from (2.2), (2.3), and (2.4), we get

$$
\begin{aligned}
\max \{ & \left\{\left(x_{5}, x_{6}, g\left(x_{5}, x_{6}\right)\right), D\left(x_{6}, x_{5}, g\left(x_{6}, x_{5}\right)\right)\right\} \\
= & \max \left\{D\left(T S x_{3}, S T x_{4}, g\left(T S x_{3}, S T x_{4}\right)\right), D\left(S T x_{4}, T S x_{3}, g\left(S T x_{4}, T S x_{3}\right)\right)\right\} \\
\leq & \max \left\{\varphi_{1}\left[\frac{1}{2}\left(D\left(x_{4}, S x_{3}, g\left(x_{4}, S x_{3}\right)\right)+D\left(x_{3}, T x_{4}, g\left(x_{3}, T x_{4}\right)\right)\right)\right],\right. \\
& \varphi_{2}\left[D\left(x_{4}, T x_{4}, g\left(x_{4}, T x_{4}\right)\right)\right], \varphi_{3}\left[D\left(x_{3}, S x_{3}, g\left(x_{3}, S x_{3}\right)\right)\right], \\
& \left.\varphi_{4}\left[D\left(x_{4}, x_{3}, g\left(x_{4}, x_{3}\right)\right)\right]\right\} \\
\leq & \max \left\{\varphi_{1}[\varphi(M)], \varphi_{2}\left[\varphi^{2}(M)\right], \varphi_{3}[\varphi(M)], \varphi_{4}[\varphi(M)]\right\} \\
\leq & \varphi^{2}(M) .
\end{aligned}
$$

In general, by induction, we get

$$
\max \left\{D\left(x_{n}, x_{n+1}, g\left(x_{n}, x_{n+1}\right)\right), D\left(x_{n+1}, x_{n}, g\left(x_{n+1}, x_{n}\right)\right)\right\} \leq \varphi^{[n / 2]}(M)
$$

for $n \geq 2$, where [ $n / 2$ ] stands for the greatest integer not exceeding $n / 2$. Since $\varphi \in \Phi$, by Singh and Meade [13, Lemma 1], it follows that $\varphi^{n}(M) \rightarrow 0$ as $n \rightarrow+\infty$ for every $M>0$. Thus, we obtain

$$
\max \left\{D\left(x_{n}, x_{n+1}, g\left(x_{n}, x_{n+1}\right)\right), D\left(x_{n+1}, x_{n}, g\left(x_{n+1}, x_{n}\right)\right)\right\} \longrightarrow 0 \text { as } n \longrightarrow \infty \text {. }
$$

Suppose that (I) does not hold. Then there exists an $\varepsilon>0$ such that for each $i \in \mathbb{N}$, there exist positive integers $n_{i}, m_{i}$, with $i \leq n_{i}<m_{i}$, satisfying

$$
\begin{gathered}
\varepsilon \leq \max \left\{D\left(x_{n_{i}}, x_{m_{i}}, g\left(x_{n_{i}}, x_{m_{i}}\right)\right), D\left(x_{m_{i}}, x_{n_{i}}, g\left(x_{m_{i}}, x_{n_{i}}\right)\right)\right\}, \\
\max \left\{D\left(x_{n_{i}}, x_{m_{i}-1}, g\left(x_{n_{i}}, x_{m_{i}-1}\right)\right), D\left(x_{m_{i}-1}, x_{n_{i}}, g\left(x_{m_{i}-1}, x_{n_{i}}\right)\right)\right\}<\varepsilon \quad \text { for } i=1,2, \ldots
\end{gathered}
$$

Set

$$
\begin{aligned}
\varepsilon_{i} & =\max \left\{D\left(x_{n_{i}}, x_{m_{i}}, g\left(x_{n_{i}}, x_{m_{i}}\right)\right), D\left(x_{m_{i}}, x_{n_{i}}, g\left(x_{m_{i}}, x_{n_{i}}\right)\right)\right\}, \\
\rho_{i} & =\max \left\{D\left(x_{i}, x_{i+1}, g\left(x_{i}, x_{i+1}\right)\right), D\left(x_{i+1}, x_{i}, g\left(x_{i+1}, x_{i}\right)\right)\right\} \quad \text { for } i=1,2, \ldots
\end{aligned}
$$


Then we have

$$
\begin{aligned}
\varepsilon \leq & \varepsilon_{i} \\
= & \max \left\{D\left(x_{n_{i}}, x_{m_{i}}, g\left(x_{n_{i}}, x_{m_{i}}\right)\right), D\left(x_{m_{i}}, x_{n_{i}}, g\left(x_{m_{i}}, x_{n_{i}}\right)\right)\right\} \\
\leq & \max \left\{D\left(x_{n_{i}}, x_{m_{i}-1}, g\left(x_{n_{i}}, x_{m_{i}-1}\right)\right), D\left(x_{m_{i}-1}, x_{n_{i}}, g\left(x_{m_{i}-1}, x_{n_{i}}\right)\right)\right\} \\
& +\max \left\{D\left(x_{m_{i}-1}, x_{m_{i}}, g\left(x_{m_{i}-1}, x_{m_{i}}\right)\right), D\left(x_{m_{i}}, x_{m_{i}-1}, g\left(x_{m_{i}}, x_{m_{i}-1}\right)\right)\right\} \\
< & \varepsilon+\rho_{m_{i}-1}, \quad i=1,2, \ldots
\end{aligned}
$$

Taking the limit as $i \rightarrow+\infty$, we get $\lim \varepsilon_{i}=\varepsilon$. On the other hand, by (2.2), (2.3), and (2.4),

$$
\begin{aligned}
\varepsilon_{i}= & \max \left\{D\left(x_{n_{i}}, x_{m_{i}}, g\left(x_{n_{i}}, x_{m_{i}}\right)\right), D\left(x_{m_{i}}, x_{n_{i}}, g\left(x_{m_{i}}, x_{n_{i}}\right)\right)\right\} \\
\leq & \max \left\{D\left(x_{n_{i}}, x_{n_{i}+1}, g\left(x_{n_{i}}, x_{n_{i}+1}\right)\right), D\left(x_{n_{i}+1}, x_{n_{i}}, g\left(x_{n_{i}+1}, x_{n_{i}}\right)\right)\right\} \\
& +\max \left\{D\left(x_{n_{i}+1}, x_{n_{i}+2}, g\left(x_{n_{i}+1}, x_{n_{i}+2}\right)\right), D\left(x_{n_{i}+2}, x_{n_{i}+1}, g\left(x_{n_{i}+2}, x_{n_{i}+1}\right)\right)\right\} \\
& +\max \left\{D\left(x_{n_{i}+2}, x_{m_{i}+2}, g\left(x_{n_{i}+2}, x_{m_{i}+2}\right)\right), D\left(x_{m_{i}+2}, x_{n_{i}+2}, g\left(x_{m_{i}+2}, x_{n_{i}+2}\right)\right)\right\} \\
& +\max \left\{D\left(x_{m_{i}+2}, x_{m_{i}+1}, g\left(x_{m_{i}+2}, x_{m_{i}+1}\right)\right), D\left(x_{m_{i}+1}, x_{m_{i}+2}, g\left(x_{m_{i}+1}, x_{m_{i}+2}\right)\right)\right\} \\
& +\max \left\{D\left(x_{m_{i}+1}, x_{m_{i}}, g\left(x_{m_{i}+1}, x_{m_{i}}\right)\right), D\left(x_{m_{i}}, x_{m_{i}+1}, g\left(x_{m_{i}}, x_{m_{i}+1}\right)\right)\right\} \\
= & \rho_{n_{i}}+\rho_{n_{i}+1}+\max \left\{D\left(x_{n_{i}+2}, x_{m_{i}+2}, g\left(x_{n_{i}+2}, x_{m_{i}+2}\right)\right),\right. \\
& \left.\quad D\left(x_{m_{i}+2}, x_{n_{i}+2}, g\left(x_{m_{i}+2}, x_{n_{i}+2}\right)\right)\right\} \\
& +\rho_{m_{i}+1}+\rho_{m_{i}} \quad \text { for } i=1,2, \ldots
\end{aligned}
$$

We will now analyze the term $\max \left\{D\left(x_{n_{i}+2}, x_{m_{i}+2}, g\left(x_{n_{i}+2}, x_{m_{i}+2}\right)\right), D\left(x_{m_{i}+2}, x_{n_{i}+2}\right.\right.$, $\left.\left.g\left(x_{m_{i}+2}, x_{n_{i}+2}\right)\right)\right\}$ based on the parity of the subscripts.

CASE 1. $n_{i}+2$ is even and $m_{i}+2$ is odd. From (2.2), (2.3), and (2.4), we have

$$
\begin{aligned}
\max \{ & \left.D\left(x_{n_{i}+2}, x_{m_{i}+2}, g\left(x_{n_{i}+2}, x_{m_{i}+2}\right)\right), D\left(x_{m_{i}+2}, x_{n_{i}+2}, g\left(x_{m_{i}+2}, x_{n_{i}+2}\right)\right)\right\} \\
= & \max \left\{D\left(S T x_{n_{i}}, T S x_{m_{i}}, g\left(S T x_{n_{i}}, T S x_{m_{i}}\right)\right), D\left(T S x_{m_{i}}, S T x_{n_{i}}, g\left(T S x_{m_{i}}, S T x_{n_{i}}\right)\right)\right\} \\
\leq & \max \left\{\varphi_{1}\left[\frac{1}{2}\left(D\left(x_{n_{i}}, S x_{m_{i}}, g\left(x_{n_{i}}, S x_{m_{i}}\right)\right)+D\left(x_{m_{i}}, T x_{n_{i}}, g\left(x_{m_{i}}, T x_{n_{i}}\right)\right)\right)\right],\right. \\
& \varphi_{2}\left[D\left(x_{n_{i}}, T x_{n_{i}}, g\left(x_{n_{i}}, T x_{n_{i}}\right)\right)\right], \varphi_{3}\left[D\left(x_{m_{i}}, S x_{m_{i}}, g\left(x_{m_{i}}, S x_{m_{i}}\right)\right)\right], \\
& \left.\varphi_{4}\left[D\left(x_{n_{i}}, x_{m_{i}}, g\left(x_{n_{i}}, x_{m_{i}}\right)\right)\right]\right\} \\
\leq & \max \left\{\varphi_{1}\left[\frac{1}{2}\left(\varepsilon_{i}+\rho_{m_{i}}+\varepsilon_{i}+\rho_{n_{i}}\right)\right], \varphi_{2}\left(\rho_{n_{i}}\right), \varphi_{3}\left(\rho_{m_{i}}\right), \varphi_{4}\left(\varepsilon_{i}\right)\right\} \\
\leq & \varphi\left(\varepsilon_{i}+\rho_{m_{i}}+\rho_{n_{i}}\right) .
\end{aligned}
$$

Therefore, we have

$$
\max \left\{D\left(x_{n_{i}+2}, x_{m_{i}+2}, g\left(x_{n_{i}+2}, x_{m_{i}+2}\right)\right), D\left(x_{m_{i}+2}, x_{n_{i}+2}, g\left(x_{m_{i}+2}, x_{n_{i}+2}\right)\right)\right\} \leq \varphi\left(k_{i}\right),
$$


where $k_{i}=\varepsilon_{i}+\rho_{m_{i}}+\rho_{n_{i}}$. Substituting (2.16) into (2.14), taking the limit as $i \rightarrow+\infty$, and using the right continuity of $\varphi$, we get

$$
\varepsilon=\lim _{i \rightarrow \infty} \varepsilon_{i} \leq \lim _{k_{i} \rightarrow \varepsilon^{+}} \varphi\left(k_{i}\right)=\varphi(\varepsilon)<\varepsilon,
$$

which is a contradiction.

CASE 2. Both $n_{i}+2$ and $m_{i}+2$ are odd. Then, we have

$$
\begin{aligned}
\max \{ & \left.D\left(x_{n_{i}+2}, x_{m_{i}+2}, g\left(x_{n_{i}+2}, x_{m_{i}+2}\right)\right), D\left(x_{m_{i}+2}, x_{n_{i}+2}, g\left(x_{m_{i}+2}, x_{n_{i}+2}\right)\right)\right\} \\
\leq & \max \left\{D\left(x_{n_{i}+2}, x_{n_{i}+1}, g\left(x_{n_{i}+2}, x_{n_{i}+1}\right)\right), D\left(x_{n_{i}+1}, x_{n_{i}+2}, g\left(x_{n_{i}+1}, x_{n_{i}+2}\right)\right)\right\} \\
& +\max \left\{D\left(x_{n_{i}+1}, x_{m_{i}+2}, g\left(x_{n_{i}+1}, x_{m_{i}+2}\right)\right), D\left(x_{m_{i}+2}, x_{n_{i}+1}, g\left(x_{m_{i}+2}, x_{n_{i}+1}\right)\right)\right\} \\
= & \rho_{n_{i}+1}+\max \left\{D\left(x_{n_{i}+1}, x_{m_{i}+2}, g\left(x_{n_{i}+1}, x_{m_{i}+2}\right)\right), D\left(x_{m_{i}+2}, x_{n_{i}+1}, g\left(x_{m_{i}+2}, x_{n_{i}+1}\right)\right)\right\} .
\end{aligned}
$$

Since $n_{i}+1$ is even and $m_{i}+2$ is odd, from Case 1 , we have

$$
\begin{aligned}
\max \{ & \left.D\left(x_{n_{i}+1}, x_{m_{i}+2}, g\left(x_{n_{i}+1}, x_{m_{i}+2}\right)\right), D\left(x_{m_{i}+2}, x_{n_{i}+1}, g\left(x_{m_{i}+2}, x_{n_{i}+1}\right)\right)\right\} \\
= & \max \left\{D\left(S T x_{n_{i}-1}, T S x_{m_{i}}, g\left(S T x_{n_{i}-1}, T S x_{m_{i}}\right)\right),\right. \\
& \left.D\left(T S x_{m_{i}}, S T x_{n_{i}-1}, g\left(T S x_{m_{i}}, S T x_{n_{i}-1}\right)\right)\right\} \\
\leq & \max \left\{\varphi_{1}\left[\frac{1}{2}\left(D\left(x_{n_{i}-1}, S x_{m_{i}}, g\left(x_{n_{i}-1}, S x_{m_{i}}\right)\right)+D\left(x_{m_{i}}, T x_{n_{i}-1}, g\left(x_{m_{i}}, T x_{n_{i}-1}\right)\right)\right)\right],\right. \\
& \varphi_{2}\left[D\left(x_{n_{i}-1}, T x_{n_{i}-1}, g\left(x_{n_{i}-1}, T x_{n_{i}-1}\right)\right)\right], \varphi_{3}\left[D\left(x_{m_{i}}, S x_{m_{i}}, g\left(x_{m_{i}}, S x_{m_{i}}\right)\right)\right], \\
& \left.\varphi_{4}\left[D\left(x_{n_{i}-1}, x_{m_{i}}, g\left(x_{n_{i}-1}, x_{m_{i}}\right)\right)\right]\right\} \\
\leq & \max \left\{\varphi_{1}\left[\frac{1}{2}\left(\rho_{n_{i}-1}+\varepsilon_{i}+\rho_{m_{i}}+\varepsilon_{i}\right)\right], \varphi_{2}\left(\rho_{n_{i}-1}\right), \varphi_{3}\left(\rho_{m_{i}}\right), \varphi_{4}\left(\rho_{n_{i}-1}+\varepsilon_{i}\right)\right\} \\
\leq & \varphi\left(\varepsilon_{i}+\rho_{m_{i}}+\rho_{n_{i}-1}\right) .
\end{aligned}
$$

Therefore, we get

$$
\max \left\{D\left(x_{n_{i}+1}, x_{m_{i}+2}, g\left(x_{n_{i}+1}, x_{m_{i}+2}\right)\right), D\left(x_{m_{i}+2}, x_{n_{i}+1}, g\left(x_{m_{i}+2}, x_{n_{i}+1}\right)\right)\right\} \leq \varphi\left(l_{i}\right),
$$

where $l_{i}=\varepsilon_{i}+\rho_{m_{i}}+\rho_{n_{i}-1}$. Hence, substituting (2.20) into (2.18), then putting (2.18) into (2.14), and taking the limit as $i \rightarrow+\infty$, we have

$$
\varepsilon=\lim _{i \rightarrow \infty} \varepsilon_{i} \leq \lim _{l_{i} \rightarrow \varepsilon^{+}} \varphi\left(l_{i}\right)=\varphi(\varepsilon)<\varepsilon,
$$

which is a contradiction. In a similar manner, we get (2.17) and (2.21) for the cases in which $n_{i}+2$ and $m_{i}+2$ are both even, and $n_{i}+2$ is odd and $m_{i}+2$ is even. That is, all cases lead to a contradiction. Therefore (I) holds. 
We claim that $\left\{x_{n}\right\}$ is $D$-Cauchy. Let $n, m, p(n<m<p)$ be any positive integers. Then, by Definition 2.1 and (2.4),

$$
\begin{aligned}
D\left(x_{n}, x_{m}, x_{p}\right) & \leq D\left(x_{n}, x_{m}, g\left(x_{n}, x_{m}\right)\right)+D\left(x_{n}, x_{p}, g\left(x_{n}, x_{m}\right)\right)+D\left(x_{m}, x_{p}, g\left(x_{n}, x_{m}\right)\right) \\
& \leq D\left(x_{n}, x_{m}, g\left(x_{n}, x_{m}\right)\right)+2 D\left(x_{n}, x_{m}, g\left(x_{n}, x_{m}\right)\right)+2 D\left(x_{m}, x_{p}, g\left(x_{m}, x_{p}\right)\right) \\
& =3 D\left(x_{n}, x_{m}, g\left(x_{n}, x_{m}\right)\right)+2 D\left(x_{m}, x_{p}, g\left(x_{m}, x_{p}\right)\right) .
\end{aligned}
$$

Since $\lim _{n \rightarrow \infty} D\left(x_{n}, x_{m}, g\left(x_{n}, x_{m}\right)\right)=0$, we have $\lim _{n \rightarrow \infty} D\left(x_{n}, x_{m}, x_{p}\right)=0$. Thus $\left\{x_{n}\right\}$ is a $D$-Cauchy sequence.

3. Main results. Now we will prove the following fixed point theorems for a complete $D$-metric space.

THeOREM 3.1. Let $(X, D)$ be a complete $D$-metric space. Let $g: X \times X \rightarrow X$ be a function and let $S$ and $T$ be self-maps on $X$ satisfying (2.2), (2.3), and (2.4) of Lemma 2.10. For any sequences $\left\{u_{n}\right\},\left\{v_{n}\right\}$ in $X$ such that $\lim _{n \rightarrow \infty} u_{n}=\alpha$ and $\lim _{n \rightarrow \infty} v_{n}=\beta, \lim _{n \rightarrow \infty} D\left(u_{n}, v_{n}\right.$, $\left.g\left(u_{n}, v_{n}\right)\right)=D(\alpha, \beta, g(\alpha, \beta))$ for some $\alpha, \beta$ in $X$.

If $S$ or $T$ is continuous, then $S$ and $T$ have a unique common fixed point.

Proof. Let the sequence $\left\{x_{n}\right\}$ be defined by $x_{0} \in X, x_{2 n+1}=T x_{2 n}$, and $x_{2 n+2}=$ $S x_{2 n+1}$ for every $n \in \mathbb{N} \cup\{0\}$. Then, by Lemma 2.10(II), it follows that $\left\{x_{n}\right\}$ is a $D$ Cauchy sequence. Since $X$ is a complete $D$-metric space, $\left\{x_{n}\right\}$ is convergent to a limit $u$ in $X$. Suppose that $S$ is continuous. Then

$$
u=\lim _{n \rightarrow \infty} x_{2 n+2}=\lim _{n \rightarrow \infty} S x_{2 n+1}=S\left(\lim _{n \rightarrow \infty} x_{2 n+1}\right)=S u .
$$

This implies that $u$ is a fixed point of $S$. From (2.2), (2.3), and (2.4), we get $D(u, S u, g(u$, $S u))=0$ and

$$
\begin{aligned}
D(u, T u, g(u, T u))= & D(u, T S u, g(u, T S u)) \\
\leq & D\left(u, x_{2 n+2}, g\left(u, x_{2 n+2}\right)\right)+D\left(S T x_{2 n}, T S u, g\left(S T x_{2 n}, T S u\right)\right) \\
\leq & D\left(u, x_{2 n+2}, g\left(u, x_{2 n+2}\right)\right) \\
& +\max \left\{\varphi_{1}\left[\frac{1}{2}\left(D\left(x_{2 n}, S u, g\left(x_{2 n}, S u\right)\right)+D\left(u, T x_{2 n}, g\left(u, T x_{2 n}\right)\right)\right)\right],\right. \\
& \varphi_{2}\left[D\left(x_{2 n}, T x_{2 n}, g\left(x_{2 n}, T x_{2 n}\right)\right)\right], \varphi_{3}[D(u, S u, g(u, S u))], \\
& \left.\varphi_{4}\left[D\left(x_{2 n}, u, g\left(x_{2 n}, u\right)\right)\right]\right\} .
\end{aligned}
$$

Taking the limit when $n$ tends to infinity, by hypothesis, we get $D(u, T u, g(u, T u))=$ 0 . Thus, we have $u=S u=T u$. Therefore, $u$ is the common fixed point of $S$ and $T$. The proof for $T$ continuous is similar. 
We will now show that $u$ is unique. Suppose that $v$ is also a common fixed point of $S$ and $T$. Then, from (2.2), (2.3), and (2.4),

$$
\begin{aligned}
\max \{D(u, v, g(u, v)), D(v, u, g(v, u))\} \\
=\max \{D(S T u, T S v, g(S T u, T S v)), D(T S v, S T u, g(T S v, S T u))\} \\
\leq \max \left\{\varphi_{1}\left[\frac{1}{2}(D(u, S v, g(u, S v))+D(v, T u, g(v, T u)))\right],\right. \\
\left.\quad \varphi_{2}[D(u, T u, g(u, T u))], \varphi_{3}[D(v, S v, g(v, S v))], \varphi_{4}[D(u, v, g(u, v))]\right\} \\
=\max \left\{\varphi_{1}\left[\frac{1}{2}(D(u, v, g(u, v))+D(v, u, g(v, u)))\right],\right. \\
\left.\quad \varphi_{2}[D(u, u, g(u, u))], \varphi_{3}[D(v, v, g(v, v))], \varphi_{4}[D(u, v, g(u, v))]\right\} \\
\leq \varphi(\max \{D(u, v, g(u, v)), D(v, u, g(v, u))\}) .
\end{aligned}
$$

We write $\max \{D(u, v, g(u, v)), D(v, u, g(v, u))\} \leq \varphi(\max \{D(u, v, g(u, v)), D(v, u$, $g(v, u))\})$, which implies that $\max \{D(u, v, g(u, v)), D(v, u, g(v, u))\}=0$, that is, $u=v$. Therefore, the common fixed point of $S$ and $T$ is unique.

REMARK 3.2. Let $X$ be a complete metric space with a metric $d$. If we take $D(x, y, z)=$ $\max \{d(x, y), d(x, z), d(y, z)\}$ and $g(x, y)=x$ for all $x, y, z \in X$, then Theorem 3.1 is Ćirić's [6, Theorem 2] which has extended a theorem of Fisher [8].

The following example shows that a $D$-metric is a proper extension of a metric $d$.

EXAMPLE 3.3. Let $d$ be a metric on $\mathbb{R}$. Define the function $\varphi: \mathbb{R} \times \mathbb{R} \rightarrow \mathbb{R}$ by $\varphi(x, y)=$ $(x-y)^{2}$ for all $x, y \in \mathbb{R}$. Then, clearly, $\varphi$ is not metric since $\varphi(2,1 / 2)>\varphi(2,1)+$ $\varphi(1,1 / 2)$. Let $G, H: \mathbb{R} \times \mathbb{R} \times \mathbb{R} \rightarrow \mathbb{R}$ be functions such that $G(x, y, z)=\max \{d(x, y)$, $d(x, z), d(y, z)\}$ and $H(x, y, z)=\max \{\varphi(x, y), \varphi(x, z), \varphi(y, z)\}$ for all $x, y, z \in \mathbb{R}$. Then, clearly, $G$ and $H$ are $D$-metric for $\mathbb{R}$. But $H$ is a $D$-metric that is a proper extension of the metric $d$. Therefore, a $D$-metric space is a proper extension of a metric space.

Corollary 3.4. Let $(X, D)$ be a complete D-metric space. Let $g: X \times X \rightarrow X$ be a function and let $S$ and $T$ be self-maps on $X$ satisfying

$$
\begin{aligned}
& \max \{D(S T x, T S y, g(S T x, T S y)), D(T S y, S T x, g(T S y, S T x))\} \\
& \leq c \cdot \max \{ \frac{1}{2}[D(x, S y, g(x, S y))+D(y, T x, g(y, T x))], \\
&D(x, T x, g(x, T x)), D(y, S y, g(y, S y)), D(x, y, g(x, y))\}
\end{aligned}
$$

for all $x, y \in X$, where $x=y$ implies $D(x, y, g(x, y))=0$ and $\max \{D(x, z, g(x, z))$, $D(x, y, g(x, z)), D(y, z, g(x, z))\} \leq D(x, y, g(x, y))+D(y, z, g(y, z))$ for all $x, y, z \in X$. 
For any sequences $\left\{u_{n}\right\},\left\{v_{n}\right\}$ in $X$ such that $\lim _{n \rightarrow \infty} u_{n}=\alpha$ and $\lim _{n \rightarrow \infty} v_{n}=\beta$, $\lim _{n \rightarrow \infty} D\left(u_{n}, v_{n}, g\left(u_{n}, v_{n}\right)\right)=D(\alpha, \beta, g(\alpha, \beta))$ for some $\alpha, \beta$ in $X$.

If $S$ or $T$ is continuous, then $S$ and $T$ have a unique common fixed point.

Proof. The proof follows by taking $\varphi_{i}(t)=c \cdot t$ with $0<c<1(i=1,2,3,4)$ in Theorem 3.1.

We will prove the following corollary using another condition instead of continuity in Theorem 3.1.

COROLlary 3.5. Let $(X, D)$ be a complete $D$-metric space. Let $g: X \times X \rightarrow X$ be a function, let $S$ and $T$ be self-maps on $X$ satisfying (2.2), (2.3), and (2.4) of Lemma 2.10, and, for each $u \in X$ with $u \neq S u$ or $u \neq T u$, let

$$
\inf \{D(x, u, g(x, u))+D(x, S x, g(x, S x))+D(y, T y, g(y, T y)): x, y \in X\}>0 .
$$

For any sequences $\left\{a_{n}\right\}$ and $\left\{b_{n}\right\}$ in $X$ such that $\lim _{n \rightarrow \infty} a_{n}=u$ and $\lim _{n \rightarrow \infty} b_{n}=v$, the following conditions hold:

(1) $\lim _{n \rightarrow \infty} D\left(a_{n}, b_{n}, g\left(a_{n}, b_{n}\right)\right)=D(u, v, g(u, v))$,

(2) $\lim _{m \rightarrow \infty} D\left(a_{n}, b_{m}, g\left(a_{n}, b_{m}\right)\right)=D\left(a_{n}, v, g\left(a_{n}, v\right)\right)$ for each $n \in \mathbb{N}$,

(3) $\lim _{m \rightarrow \infty} D\left(b_{m}, a_{n}, g\left(b_{m}, a_{n}\right)\right)=D\left(v, a_{n}, g\left(v, a_{n}\right)\right)$ for each $n \in \mathbb{N}$.

Then $S$ and $T$ have a unique common fixed point.

Proof. From Lemma 2.10(I) and (II), the sequence $\left\{x_{n}\right\}$ defined by $x_{0} \in X, x_{2 n+1}=$ $T x_{2 n}$, and $x_{2 n+2}=S x_{2 n+1}$ for every $x \in \mathbb{N} \cup\{0\}$ is a $D$-Cauchy sequence. Since $X$ is a complete $D$-metric space, there exists $u \in X$ such that $\left\{x_{n}\right\}$ converges to $u$. Then we have

$$
\begin{aligned}
& D\left(x_{2 n+1}, x_{2 m+2}, g\left(x_{2 n+1}, x_{2 m+2}\right)\right) \\
&=D\left(T S x_{2 n-1}, S T x_{2 m}, g\left(T S x_{2 n-1}, S T x_{2 m}\right)\right) \\
& \leq \max \{ \varphi_{1}\left[\frac{1}{2}\left(D\left(x_{2 m}, S x_{2 n-1}, g\left(x_{2 m}, S x_{2 n-1}\right)\right)+D\left(x_{2 n-1}, T x_{2 m}, g\left(x_{2 n-1}, T x_{2 m}\right)\right)\right)\right], \\
& \varphi_{2}\left[D\left(x_{2 m}, T x_{2 m}, g\left(x_{2 m}, T x_{2 m}\right)\right)\right], \varphi_{3}\left[D\left(x_{2 n-1}, S x_{2 n-1}, g\left(x_{2 n-1}, S x_{2 n-1}\right)\right)\right], \\
&\left.\varphi_{4}\left[D\left(x_{2 m}, x_{2 n-1}, g\left(x_{2 m}, x_{2 n-1}\right)\right)\right]\right\} \\
& \leq \max \{ \varphi_{1}\left[\frac{1}{2}\left(D\left(x_{2 m}, x_{2 n}, g\left(x_{2 m}, x_{2 n}\right)\right)+D\left(x_{2 n-1}, x_{2 m+1}, g\left(x_{2 n-1}, x_{2 m+1}\right)\right)\right)\right], \\
& \varphi_{2}\left[D\left(x_{2 m}, x_{2 m+1}, g\left(x_{2 m}, x_{2 m+1}\right)\right)\right], \varphi_{3}\left[D\left(x_{2 n-1}, x_{2 n}, g\left(x_{2 n-1}, x_{2 n}\right)\right)\right], \\
&\left.\varphi_{4}\left[D\left(x_{2 m}, x_{2 n-1}, g\left(x_{2 m}, x_{2 n-1}\right)\right)\right]\right\} .
\end{aligned}
$$

Thus, we obtain $\lim _{n \rightarrow \infty} D\left(x_{2 n+1}, u, g\left(x_{2 n+1}, u\right)\right)=0$. Assume that $u \neq S u$ or $u \neq T u$. 
Then, by hypothesis, we have

$$
\begin{aligned}
0<\inf \{ & D(x, u, g(x, u))+D(x, S x, g(x, S x))+D(y, T y, g(y, T y)): x, y \in X\} \\
\leq & \inf \left\{D\left(x_{2 n+1}, u, g\left(x_{2 n+1}, u\right)\right)+D\left(x_{2 n+1}, S x_{2 n+1}, g\left(x_{2 n+1}, S x_{2 n+1}\right)\right)\right. \\
& \left.+D\left(x_{2 n+2}, T x_{2 n+2}, g\left(x_{2 n+2}, T x_{2 n+2}\right)\right): n \in \mathbb{N}\right\} \\
= & \inf \left\{D\left(x_{2 n+1}, u, g\left(x_{2 n+1}, u\right)\right)+D\left(x_{2 n+1}, x_{2 n+2}, g\left(x_{2 n+1}, x_{2 n+2}\right)\right)\right. \\
\quad & \left.\quad D\left(x_{2 n+2}, x_{2 n+3}, g\left(x_{2 n+2}, x_{2 n+3}\right)\right): n \in \mathbb{N}\right\} \\
= & 0 .
\end{aligned}
$$

This is a contradiction. Therefore, we have $u=S u=T u$.

On the other hand, we can prove the existence of a unique common fixed point of $S$ and $T$ by a method similar to that of Theorem 3.1.

ACKNOWLEDGMENT. This work was supported by Korea Research Foundation Grant (KRF-2003-015-C00039).

\section{REFERENCES}

[1] D. W. Boyd and J. S. W. Wong, On nonlinear contractions, Proc. Amer. Math. Soc. 20 (1969), 458-464.

[2] J. L. C. Camargo, An application of a fixed-point theorem of D. W. Boyd and J. S. W. Wong, Rev. Mat. Estatíst. 6 (1988), 25-29.

[3] S. K. Chatterjea, Applications of an extension of a theorem of Fisher on common fixed point, Pure Math. Manuscript 6 (1987), 35-38.

[4] Lj. B. Ćirić, A generalization of Banach's contraction principle, Proc. Amer. Math. Soc. 45 (1974), 267-273.

[5] _ A new fixed-point theorem for contractive mappings, Publ. Inst. Math. (Beograd) (N.S.) 30(44) (1981), 25-27.

[6] _ Common fixed points of nonlinear contractions, Acta Math. Hungar. 80 (1998), no. 12, 31-38.

[7] B. C. Dhage, Generalised metric spaces and mappings with fixed point, Bull. Calcutta Math. Soc. 84 (1992), no. 4, 329-336.

[8] B. Fisher, Some results on fixed points, Acta Math. Acad. Sci. Hungar. 33 (1979), no. 3-4, 289-292.

[9] O. Hadžić, Some applications of a common fixed point theorem, Studia Univ. Babeş-Bolyai Math. 28 (1983), 67-74.

[10] A. Meir and E. Keeler, A theorem on contraction mappings, J. Math. Anal. Appl. 28 (1969), 326-329.

[11] H. K. Pathak, Fixed point theorems for weak compatible multi-valued and single-valued mappings, Acta Math. Hungar. 67 (1995), no. 1-2, 69-78.

[12] B. E. Rhoades, A comparison of various definitions of contractive mappings, Trans. Amer. Math. Soc. 226 (1977), 257-290.

[13] S. P. Singh and B. A. Meade, On common fixed point theorems, Bull. Austral. Math. Soc. 16 (1977), no. 1, 49-53.

[14] M. Turinici, Multivalued contractions and applications to functional-differential equations, Acta Math. Acad. Sci. Hungar. 37 (1981), no. 1-3, 147-151. 
[15] A. Wieczorek, Applications of fixed-point theorems in game theory and mathematical economics, Wiadom. Mat. 28 (1988), no. 1, 25-34.

Hee Soo Park: Department of Applied Mathematics, Changwon National University, Changwon 641-773, Korea

E-mail address: pheesoo@changwon.ac.kr

Jeong Sheok Ume: Department of Applied Mathematics, Changwon National University, Changwon 641-773, Korea

E-mail address: jsume@changwon.ac. kr 


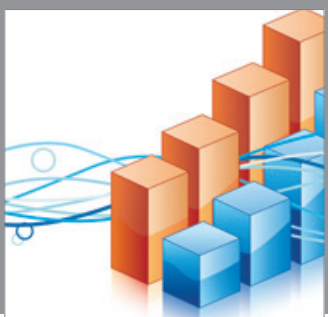

Advances in

Operations Research

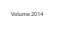

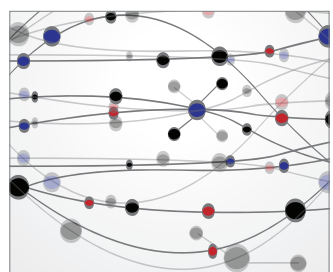

\section{The Scientific} World Journal
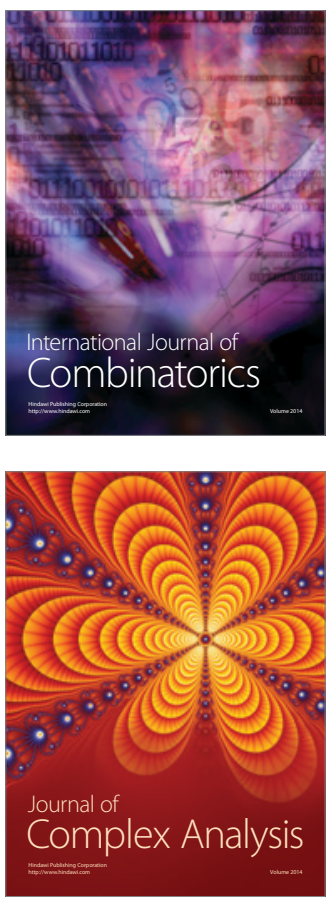

International Journal of

Mathematics and

Mathematical

Sciences
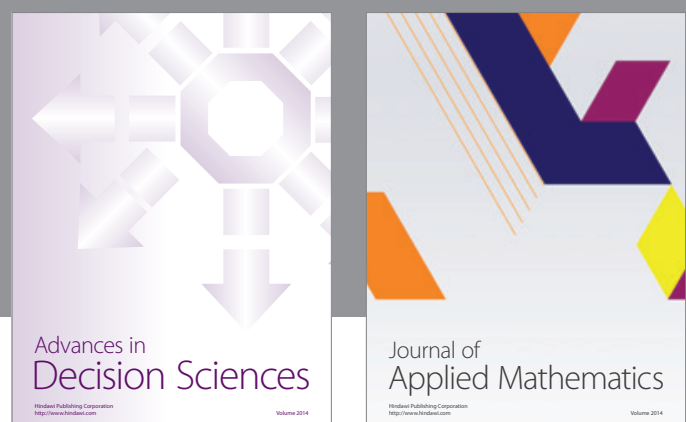

Journal of

Applied Mathematics
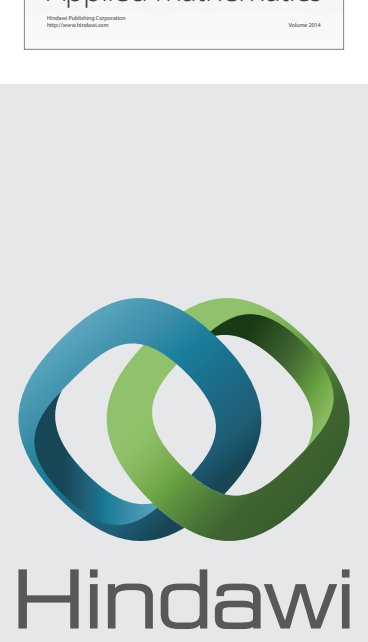

Submit your manuscripts at http://www.hindawi.com
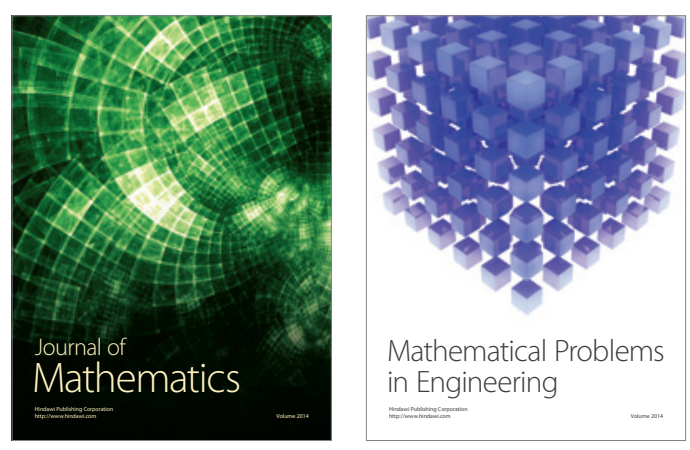

Mathematical Problems in Engineering
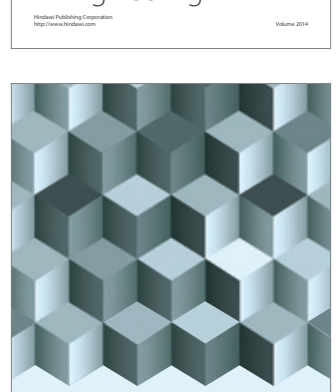

Journal of

Function Spaces
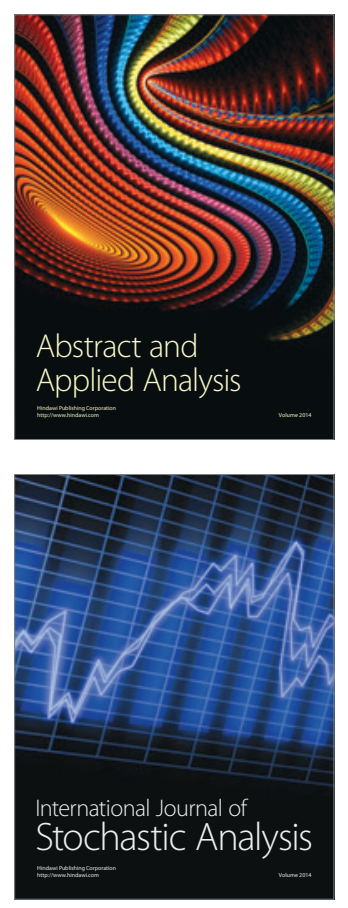

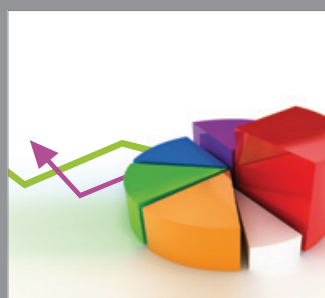

ournal of

Probability and Statistics

Promensencen
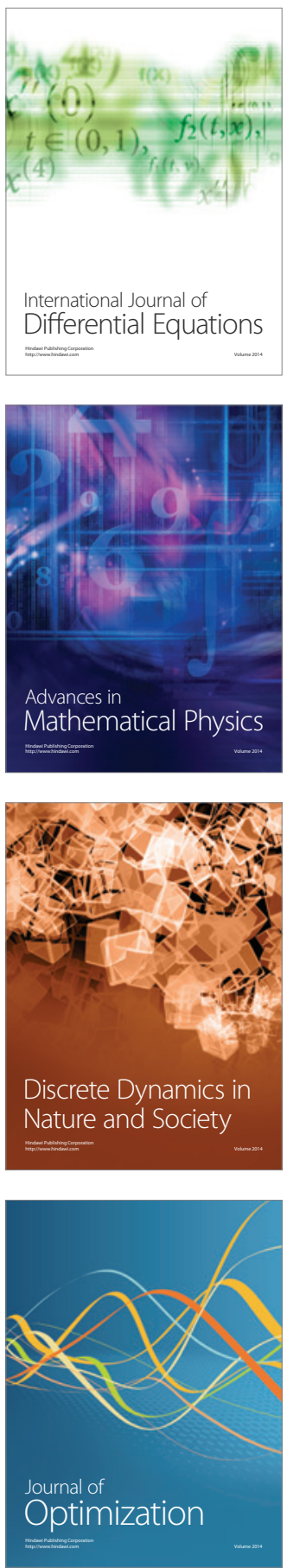